Article

\title{
Independent Component Analysis Data Processing Framework for Polarimetric Synthetic Aperture Radar Images
}

\author{
Gabriel Vasile ${ }^{1}[1$ * \\ 1 Grenoble-Image-sPeach-Signal-Automatics Lab, Univ. Grenoble Alpes, CNRS / Grenoble INP, Grenoble, \\ France; gabriel.vasile@grenoble-inp.fr \\ + This paper is an extended version of our paper published in IEEE International Geoscience and Remote \\ Sensing Symposium.
}

\begin{abstract}
The Independent Component Analysis (ICA) has been recently introduced as a reliable alternative to identify canonical scattering mechanisms within Polarimetric Synthetic Aperture Radar (PolSAR) images. This manuscript addresses two important aspects when applying such methods on real data, namely speckle filtering and statistical classification with ICA. A novel PolSAR data processing framework is introduced by adjusting the Lee's sigma filter to the particular nature of the Touzi's polarimetric decomposition. In its current form, it allows the use of the ICA mixing matrix in the derived speckle filter. An extension of the Fromont at al. iterative segmentation is introduced, equally. This proposed framework is tested using P band airborne PolSAR data acquired for the ESA campaign TropiSAR campaign.
\end{abstract}

Keywords: Independent Component Analysis; PolSAR; speckle filtering; statistical classification.

\section{Introduction}

Polarimetric target decomposition is a PolSAR image interpretation technique that relies on the analysis of the interaction between the illuminated area and the transmitted waveform, considering each polarimetric state of the latter. More specifically, it enables the description of an image cell as a sum of canonical scattering mechanisms (also called as target vectors) making it more intuitive to understand the behavior of the clutter and therefore to analyze it [1-3].

Polarimetric target decompositions are mainly classified in coherent, if their interest lies on the scattering matrix analysis for each resolution cell, like the ones proposed by Cameron [4], or incoherent, if they are based on a statistical analysis of neighboring pixels. Incoherent target decomposition (ICTD) theory assumes that the scattering process in most natural media is a combination of coherent speckle noise [5] and random vector scattering effects [6,7]. Therefore, a stochastic approach is required and the concept of average or dominant scattering mechanisms is associated to each imaging cell [6]. Most methods described in the literature focus on the Hermitian, semidefinite positive coherence or covariance matrix [6]. Nevertheless, the investigation of higher order moments has recently sparked great interest of the SAR community, introducing supplementary information to the clutter analysis and consequently leading to new ICTD approaches [8], [9], [10].

The combined use of the eigenvector approach with Cloude and Pottier's parametrization gave rise to one of the most employed and most traditional classification schemes in PolSAR data analysis, the $H / \alpha$ feature space [11]. The entropy, $H$, measures the degree of randomness of the scattering phenomenon, given as a function of the eigenvalues of the coherence matrix. Each eigenvector correspond to a scattering mechanism within the image cell and therefore each one will provide a 
different $\alpha$ angle. The authors in [11] state that the best estimate of such parameter to represent the image cell is an weighted average based on the eigenvalues of the coherence matrix.

The parameters $H$ and $\alpha$ are plotted in a plane, originating the so called $H / \alpha$ feature space. Upon the introduction of the aforementioned method, Cloude and Pottier suggested the partitioning of the plane in nine regions, based on the polarimetric behavior of known type of natural phenomenons. Therefore, once the $H$ and the average $\alpha$ parameters are extracted from the target polarimetric signature, it is straightforward to classify it as one of the corresponding type of scattering mechanisms. Many works are based on such method, from geophysical parameters inversion algorithms (in varied regions from the globe) to detection and classification algorithms. Having a remarkable correspondence to ground truth, the usage of this unsupervised technique has had very few improvements since its conception. Nevertheless, there is an important remark still uncovered related to this method. The orthogonality constraint of the eigenvectors of the coherence matrix generates unfeasible regions in the plane. It is important to highlight that these regions are mathematically, and not physically, unfeasible.

In [8], a new strategy to polarimetric target decomposition was presented by incorporating the Independent Component Analysis (ICA) as an alternative to identify the canonical scattering mechanisms within an image cell. The ICA is a blind source separation technique based on higher order statistical moments and cumulants whose utility has already been explored in many different research areas, such as wireless communications, data compression and brain imaging applications. The results presented in [8] proved it to be a very promising area in polarimetry, mainly when non-Gaussian heterogeneous clutters (inherent to high resolution SAR systems) are under study. The theoretical potential in estimating similar entropy and first component, when compared to traditional eigenvector decomposition, but rather a second most dominant component independent with respect to the first one and unconstrained by the orthogonality introduces an alternative way of physically interpreting a polarimetric SAR image. In [9], the ability of ICA to correctly identify clutters composed by non-orthogonal type of scatters is further investigated, as well as its performance under a sliding window approach, enabling a more accurate comparison to the results obtained with the eigenvector based approach.

Within this context, this paper is synthesizing the results presented in $[12,13]$ in order to propose an unified PolSAR data processing and analysis framework for the ICA based ICTD. The paper is structured as follows. Section 2 introduces the context of ICA-ICTD, Section 3 describes the proposed ICA-ICTD data processing framework, while Section 4 presents some qualitative and quantitative performance assessment. Section 5 concludes the paper.

\section{ICA based ICTD}

Polarimetric SAR images can be used for several applications, for example for land cover classification. The ICA based ICTD decomposition is targeting the estimation of the mixing matrix A (Eq. 1). There are several criteria for determining the elements of $\mathbf{A}$ in order to ensure the mutual independence of the sources in $\mathbf{s}$. The common factor for all the applied methods is the assumption that at most one of the sources is Gaussian.

$$
\mathbf{k}^{c}(i, j)=\left[\begin{array}{lll}
A_{11}^{c} & A_{12}^{c} & A_{13}^{c} \\
A_{21}^{c} & A_{22}^{c} & A_{23}^{c} \\
A_{31}^{c} & A_{32}^{c} & A_{33}^{c}
\end{array}\right] \cdot\left[\begin{array}{l}
s_{1}^{c}(i, j) \\
s_{2}^{c}(i, j) \\
s_{3}^{c}(i, j)
\end{array}\right]=\mathbf{A}^{c} \mathbf{s}^{c}(i, j)
$$

The selected Complex Fast-ICA algorithm is based on a bottom-up approach: emphasizing the non-gaussanity of the sources by maximizing an arbitrary nonlinear contrast function (Eq. 2) whose extrema coincides with the independent component.

$$
J_{G}(\mathbf{w})=\mathbf{E}\left\{\mathbf{G}\left(\left|\mathbf{w}^{\mathbf{H}} \mathbf{x}\right|^{2}\right)\right\}
$$


The performances of the algorithm depend strongly on the choice of the nonlinear function $G(y)$, which is supposed to be suited to the particular application. Therefore, here we have used the kurtosis criterion in deriving independent target vectors:

$$
G_{1}(y)=\frac{1}{2} y^{2}
$$

In this case, the contrast functions becomes essentially a measure of the fourth statistical moment of the source. As its value in case of the Gaussian variable equals zero, by maximizing the kurtosis of each of the sources, we ensure their independence.

The result of the incoherent target decomposition is the set of target vectors representing elementary scatterers and a set of scalars, providing their proportion in the total scattering. In our case, the target vectors of the independent scatterers are the columns of the estimated mixing matrix $\mathbf{A}=\mathbf{W}^{\mathbf{H}}$. The contribution to the total backscattering $(m)$ is computed as a square root of the maximal eigenvalue of the derived Graves matrix.

Being based on Kennaugh-Huynen condiagonalization projected onto the Pauli basis, the Touzi's Target Scattering Vector Model (TSVM) [1] allows parametrization of the target vector in terms of rotation angle $(\psi)$, maximum amplitude $(m)$, target helicity $\left(\tau_{m}\right)$, symmetric scattering type magnitude $\left(\alpha_{s}\right)$ and symmetric scattering type phase $\left(\Phi_{\alpha_{s}}\right)$, among which the last four are roll-invariant:

$$
\mathbf{k}=m|\mathbf{k}|_{m} e^{j \Phi_{s}}\left[\begin{array}{ccc}
1 & 0 & 0 \\
0 & \cos 2 \psi & -\sin 2 \psi \\
0 & \sin 2 \psi & \cos 2 \psi
\end{array}\right]\left[\begin{array}{c}
\cos \alpha_{s} \cos 2 \tau_{m} \\
\sin \alpha_{s} e^{j \Phi_{\alpha_{s}}} \\
-j \cos \alpha_{s} \sin 2 \tau_{m}
\end{array}\right] .
$$

Using these parameters, it is eventually possible to represent the obtained independent target vectors on either symmetric or non-symmetric target Poincaré sphere. In our case, they do not necessarily form an orthogonal basis.

In general, the limiting curves on the $\mathrm{H} / \alpha$ plane reduce the analysis of high entropy type of targets in a more limiting way than the analysis of low entropy ones. Even though polarimetry is a better suited tool to analyze low entropy type of targets, the ability to correctly estimate the parameters that describe the illuminated scatters is crucial. Once again, it is important to highlight that these regions are mathematically, and not physically, unfeasible [10].

Previous results [9] demonstrate the $11 \times 11$ spatial neighborhood is well suited for the estimation of the Touzi's TSVM parameters from the ICA mixing matrix (using Single Look Complex target vectors). In the present work, our focus relies on addressing the following issues: to propose speckle filtering in ICA-ICTD for better preservation of spatial features and to introduce an appropriate statistical classification algorithm.

\section{ICA-ICTD speckle filtering}

One of the most powerful and widely used speckle filters in PolSAR is the Lee's sigma filter, with its recent improvement proposed in [14]. In this paper, we propose to use the same algorithm for deriving the adaptive Minimum Mean Square Error (MMSE) coefficient $b$ from Eq. (6) in [14]. Further on, the following modifications are operated.

Firstly, instead of using each intensity image separately, we propose to use the Polarimetric Whitening Filter for taking into account simultaneously all the PolSAR channels. To do that, one good candidate is the texture parameter $\tau$ from [15] computed in each pixel:

$$
\widehat{\tau}=\mathbf{k}^{\dagger}[\widehat{M}]_{F P}^{-1} \mathbf{k}
$$

where $[\widehat{M}]_{F P}^{-1}$ is the Fixed Point polarimetric covariance matrix estimated over the centred boxcar neighbourhood and $\mathbf{k}$ the corresponding target vector. 
Secondly, the MMSE filter is modified in each pixel of the PolSAR span image as:

$$
\widehat{s}=(1-b) \widehat{\sigma}_{0}+b \widehat{\tau}
$$

where $\widehat{\sigma_{0}}=\frac{\mathbf{k}^{\dagger}[\widehat{M}]_{F P}^{-1} \mathbf{k}}{\mathbf{k}^{\dagger}[\widehat{T}]_{S C M}^{-1} \mathbf{k}}$ and $[\widehat{T}]_{S C M}$ the sample covariance matrix.

Thirdly, the strong deterministic scatterers, previously detected by the 98th percentile as in [14], are updating the values of the MMSE coefficient $b$ to 1 . For each pixel, the values of this coefficient is stored and will be used later for filtering the corresponding TSVM parameters. One can notice that, at this stage, we have available the filtered PolSAR span and the MMSE coefficient images.

When adapting the Lee's sigma filter to the ICA-ICTD, the most important constraint is the non-orthogonality of the ICA derived backscattering mechanisms. In [14], the MMSE filter from Eq. (6) has been directly applied for the multi-look PolSAR covariance matrix. This holds because two Sample Covariance estimates can be averaged, but this is not true for the ICA derived mixing matrices.

Instead of taking the average of the two covariance matrices, the mixing matrices barycenter could be addressed. The implementation of such approach is not straightforward, since the task of estimating matrix geometric means is a complex subject. The concept of geometric mean for more than two matrices has only been fully defined recently [16], powered by the association of the geometric mean of two positive definite matrices, $[M]_{1}$ and $[M]_{2}$, as the midpoint of the geodesic (with respect to a natural Riemannian metric) joining $[M]_{1}$ and $[M]_{2}$. While the derived theory is valid when addressing positive definite matrices (which is the case of the covariance matrices), it does not hold for the mixing matrices estimated with ICA, which are not necessarily positive definite.

Nevertheless, the solution relies on the rotational invariance of the Touzi's TSVM decomposition. From [1], one can observe that the rotation invariant scattering vector has the following form:

$$
\overrightarrow{\mathbf{v}}^{\text {orient-inv }}=\mu\left[\begin{array}{c}
\cos \alpha_{s} \cos \left(2 \tau_{m}\right) \\
\sin \alpha_{s} \rho^{j \Phi_{\alpha_{s}}} \\
-j \cos \alpha_{s} \sin \left(2 \tau_{m}\right)
\end{array}\right],
$$

where the four roll-invariant parameters $\alpha_{s}$ (symmetric scattering type magnitude), $\Phi_{\alpha_{s}}$ (symmetric scattering type phase), $\tau_{m}$ (helicity), and $\mu$ (maximum amplitude return) are necessary for an unambiguous description of the corresponding PolSAR scattering mechanism. As described in [1], $\overrightarrow{\mathbf{v}}$ orient-inv is obtained by constructing the Graves power matrix and performing the con-diagonalization from [17] followed by the Huynen desying.

In each pixel, we propose to filter independently each rotation invariant scattering vector $\overrightarrow{\mathbf{v}}_{i}^{\text {orient-inv }}$ retrieved from the columns of the ICA derived mixing matrix ( $i=\overline{1,3}$ for monostatic

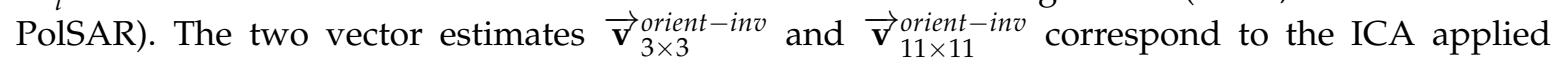
independently on $3 \times 3$ and respectively $11 \times 11$ boxcar neighborhoods:

$$
\overrightarrow{\mathbf{v}} \text { orient }-i n v=(1-b) \overrightarrow{\mathbf{v}}_{11 \times 11}^{\text {orient-inv }}+b \overrightarrow{\mathbf{v}}_{3 \times 3}^{\text {orient-inv }} .
$$

Finally, the Cloude and Pottier entropy can be evaluated using the maximum amplitude returns $\mu_{i}$, while the Cloude and Pottier $\alpha$ is equivalent to Touzi's $\alpha_{s}$ for symmetric scattering [1]. The flowchart of the proposed speckle filtering algorithm is summarized in Fig. 1.

\subsection{ICA-ICTD classification}

In this section, we introduce an extension of the statistical classification algorithm presented in [18] for direct application to ICA based ICTD.

One possibility would be to employ directly the mixing matrices from [8] and compute the local barycenter. However, the derived theory from [18] is valid with positive definite matrices, only. This does not hold for the ICA mixing matrices, which are not necessarily positive definite. The proposed 


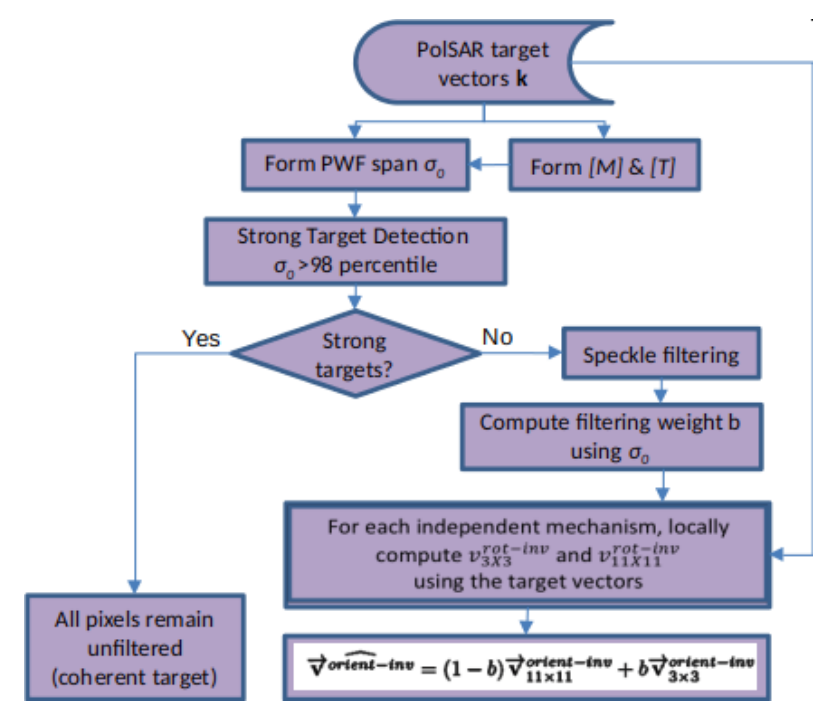

Figure 1. Flowchart of the proposed ICA-ICTD speckle filtering.

solution consists in employing each ICA derived dominant scattering mechanism and form either three $3 \times 1$ or one $9 \times 1$ input complex random vectors.

The only restriction would be that the use of the ICA scattering mechanisms are not directly rotational invariant. As introduced previously, we propose once again to use the rotation invariant scattering vectors from Eq. 7. The flowchart of the derived classification algorithm is illustrated in Fig. 2.

\section{PolSAR experimental results}

The PolSAR dataset was acquired by the French Aerospace Lab (ONERA), in 2009, over the French Guiana, in the frame of the European Space Agency (ESA) campaign TropiSAR. Fig. 3-(a),(b) shows the classification results obtained using the $\mathrm{H} / \alpha$ eigenvector decomposition (PCA) and $\mathrm{H} / \alpha$ ICA decomposition, respectively. One can notice that similar mechanisms were detected, mostly in zone 2, with significantly less bias in the ICA based ICTD.

The PolSAR speckle filtering results are illustrated in Fig. 4-(a),(b). In this paper, a decimation by a factor 2 has been applied both in range and azimuth (equivalent to a 4-look covariance matrix). As expected, the proposed MMSE filter outperforms the boxcar filter in terms of spatial resolution preservation.

Fig. 5-(e),(f),(g),(h) shows the Touzi's roll invariant TSVM parameters computed by ICA MMSE speckle filtering, as compared to the ones obtained by applying the boxcar filter and PCA from Fig. $5-(a),(b),(c),(d)$.

We propose to analyze the results by representing the derived TSVM parameters of both symmetric (helicity equal 0 ) and non-symmetric (symmetric scattering type phase equal 0 ) targets on the Poincaré sphere It can be observed in Fig. 6-(a),(b) that the second and the third most dominant mechanism (represented in blue and green, respectively) occupy different position onto the sphere, thus meaning that the non-orthogonality of ICA will produce new mechanisms, indeed.

After speckle filtering, the K-Means type iterative segmentation is applied using the Riemannian mean [19]. The maximum number of iterations has been set to $N=10$. One can observe, in Fig. $7-(a),(b),(c)$, the barycenter evolution in the $\mathrm{H} / \alpha$ plane for the three ICA scattering mechanisms: the entropy is considerably decreasing with the number of iterations indicating a better statistical clustering, while the $\alpha$ angle is moving from anisotropic particle to dipole scattering (which corresponds to forested areas at $\mathrm{P}$ band). 


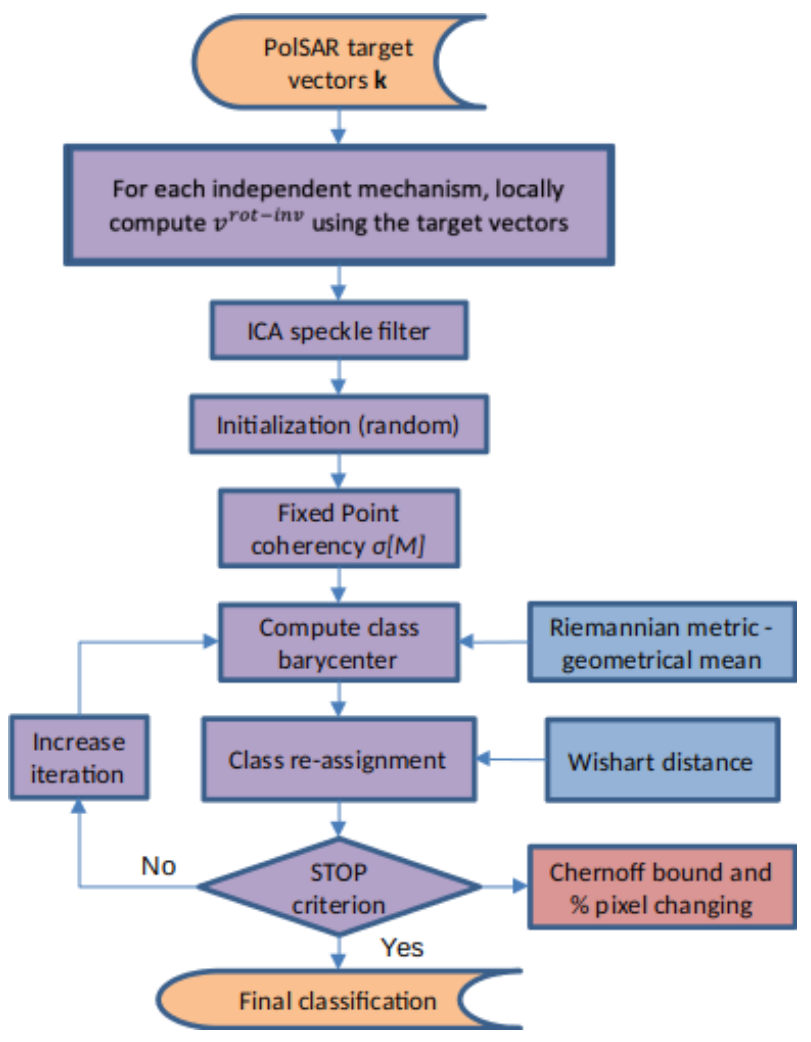

Figure 2. Flowchart of the proposed ICA-ICTD classification.

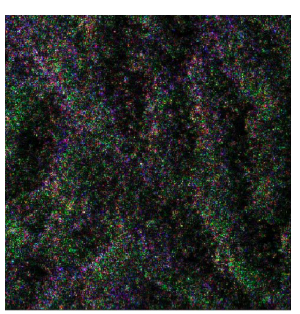

(a)

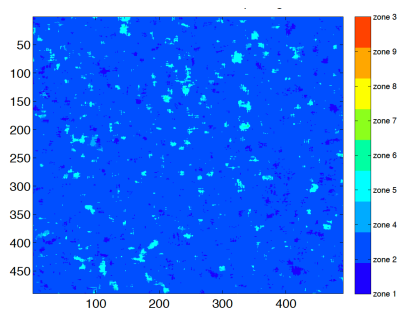

(b)

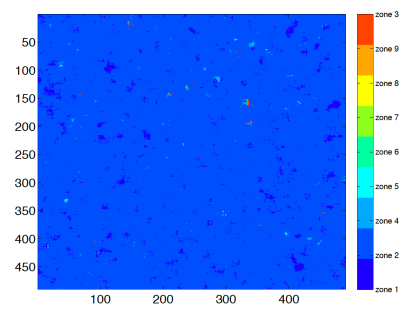

(c)

Figure 3. Paracou P-band airborne dataset: (a) initial RGB representation in Pauli basis, $H / \alpha$ classification map (b) PCA and (c) ICA. 


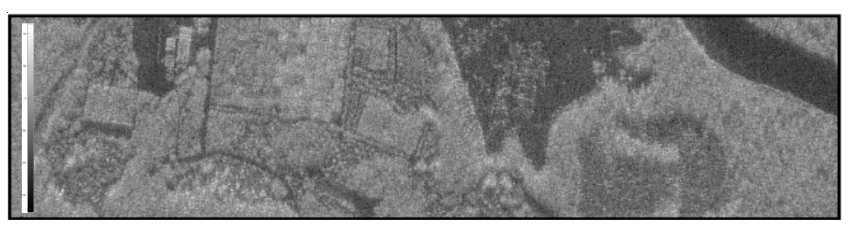

(a)

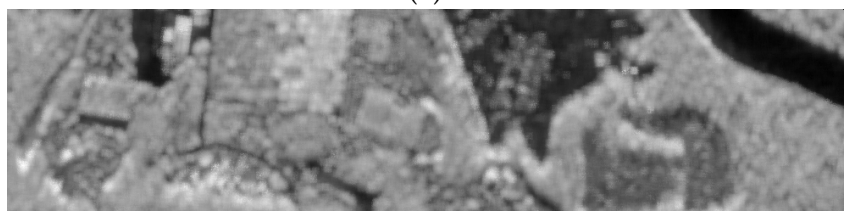

(b)

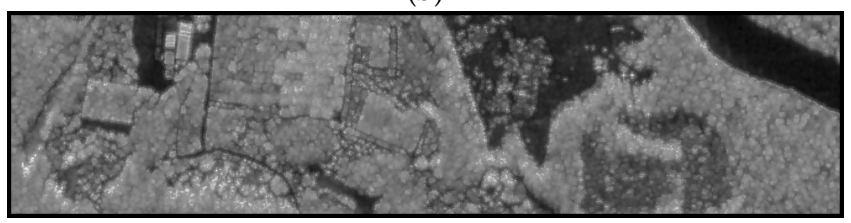

(c)

Figure 4. Paracou P-band airborne dataset, speckle filtering results, filtered PolSAR span image (in dB) : (a) initial, (b) boxcar and (c) MMSE.

Fig. 8 illustrates the Chernoff bound and the percentage of pixels moving to another class at each iteration. Both indicators are indicating the convergence of the proposed classification algorithm, while revealing that the optimal number of iteration for the proposed classification algorithm is 5 .

Finally, the final statistical classification result is presented in Fig. 9.

\section{Conclusion}

This paper proposed a novel data processing framework for PolSAR images. In the context of ICA-ICTD, the Lee's sigma filter has been adapted to the specific nature of the polarimetric mixing matrix. By applying the MMSE filter on each of the ICA derived rotation invariant scattering vectors, we demonstrated the spatial resolution can be better preserved with respect to the conventional PolSAR boxcar speckle filter.

Based on Formont's iterative statistical classifier, the proposed "theoretically clean" framework is able to process PolSAR data, when the ICA-ICTD is applied. It has been tested using P band airborne PolSAR data over forested areas.

At this stage, further developments are required in order to evolve the proposed algorithm towards an user friendly more intuitive application.

Funding: This research was funded by the National Center for Scientific Research (CNRS), France.

Acknowledgments: Thanks to the French Aerospace Lab for providing the PolSAR data.

Conflicts of Interest: The authors declare no conflict of interest. The funders had no role in the design of the study; in the collection, analyses, or interpretation of data; in the writing of the manuscript, or in the decision to publish the results.

\section{Abbreviations}

The following abbreviations are used in this manuscript: 


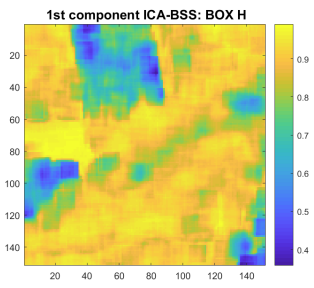

(a)

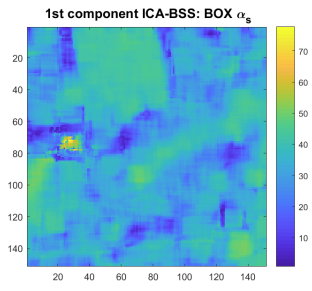

(b)

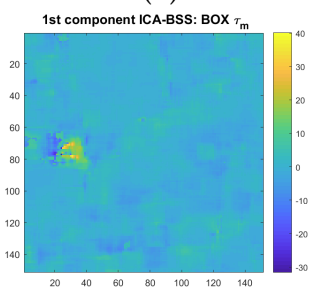

(c)

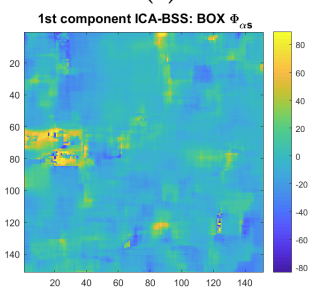

(d)

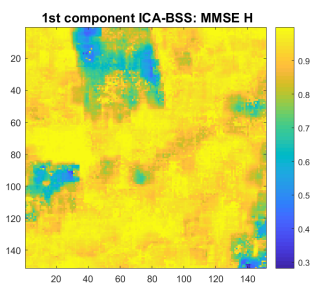

(e)

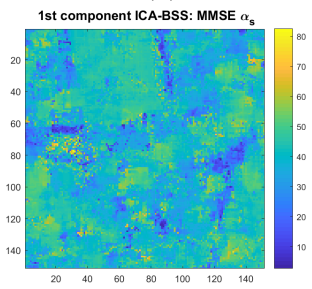

(f)

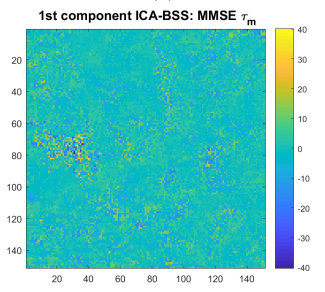

(g)

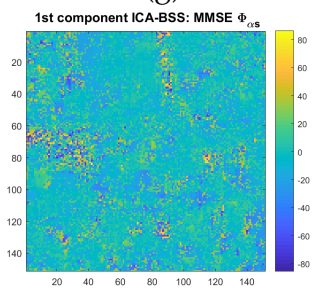

(h)

Figure 5. Paracou P-band airborne dataset, entropy, symmetric scattering type magnitude, helicity and, respectively, symmetric scattering type phase: (a), (b), (c), (d) TSVM parameters after PCA boxcar speckle filtering and (e), (f), (g), (h) TSVM parameters after ICA MMSE speckle filtering. 


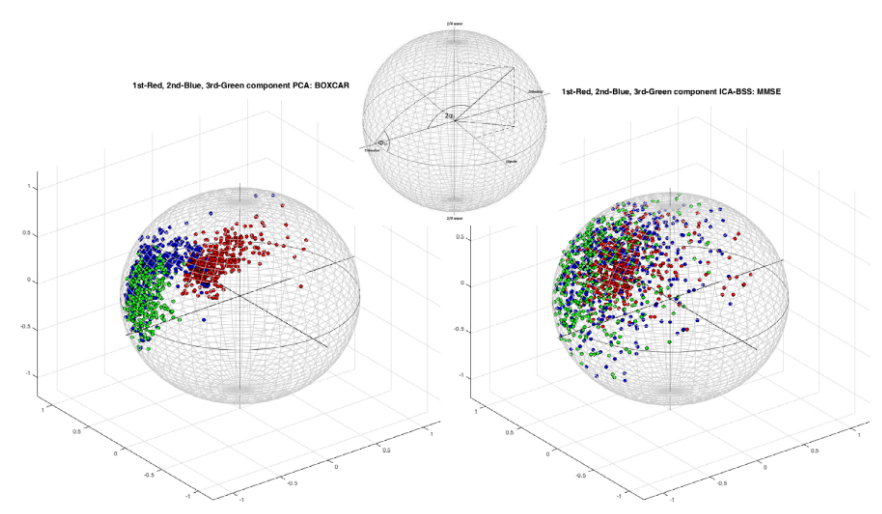

(a)

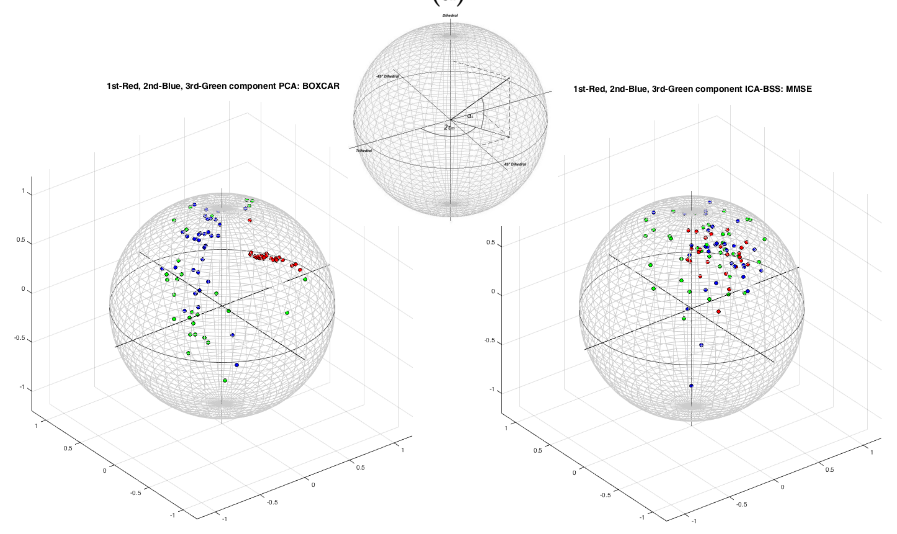

(b)

Figure 6. Paracou P-band airborne dataset, Poincaré sphere representation of symmetric targets, Boxcar PCA and MMSE ICA: (a) symmetric targets $\left(\tau_{m}=0\right)$, (b) non-symmetric targets $\left(\Phi_{\alpha_{s}}=0\right)$. 


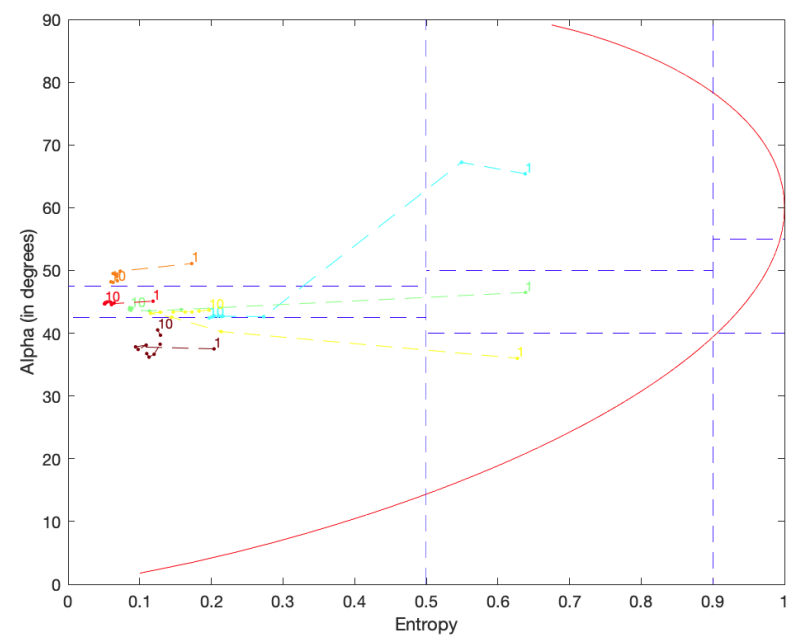

(a)

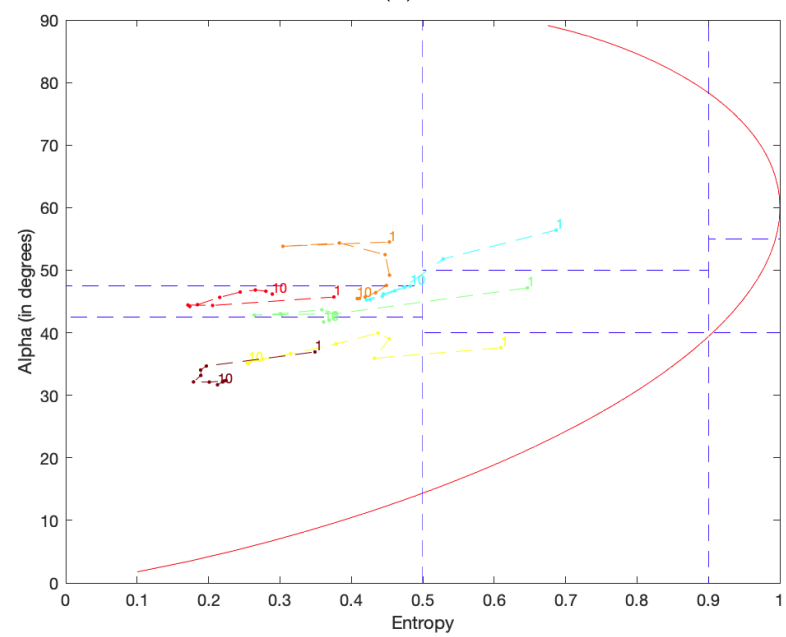

(b)

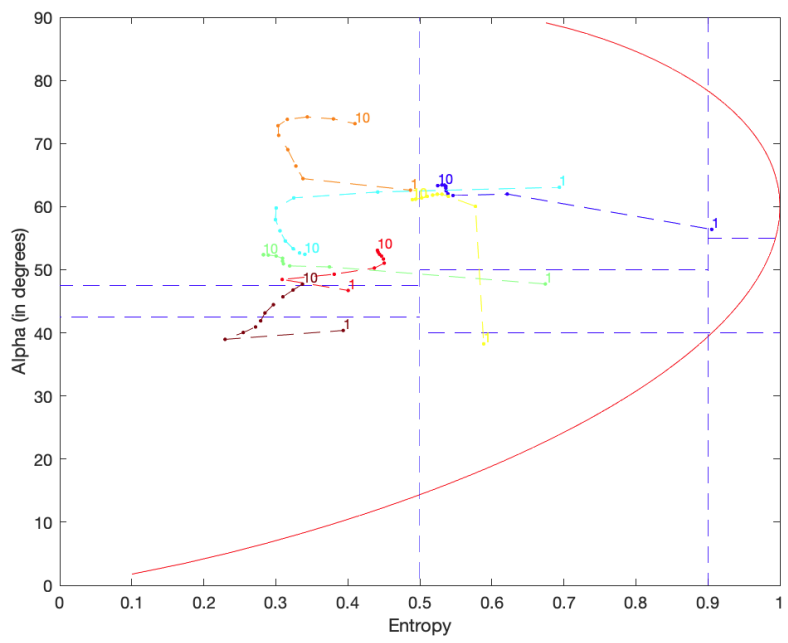

(c)

Figure 7. Paracou P-band airborne dataset: $H / \alpha$ plane moving centers: (a) first component, (b) second component, (c) third component. 


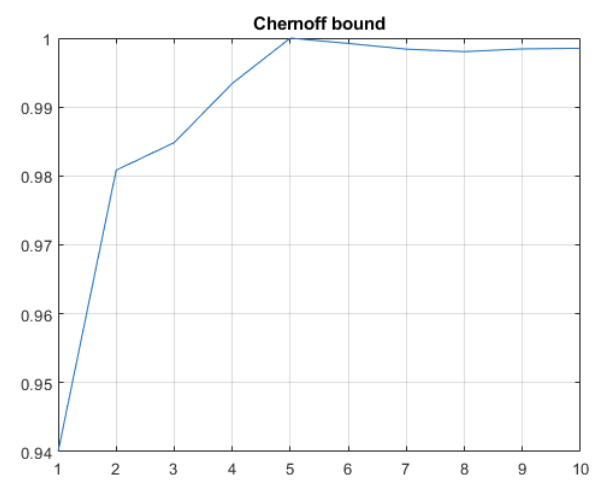

(a)

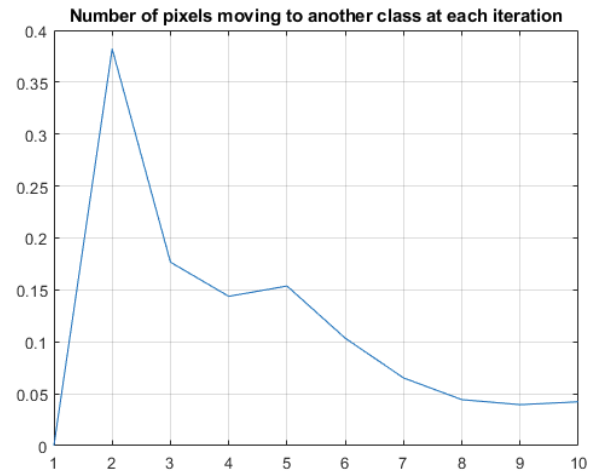

(b)

Figure 8. Paracou P-band airborne dataset: (a) Chernoff bound and (b) percentage of pixels moving to another class at each iteration.

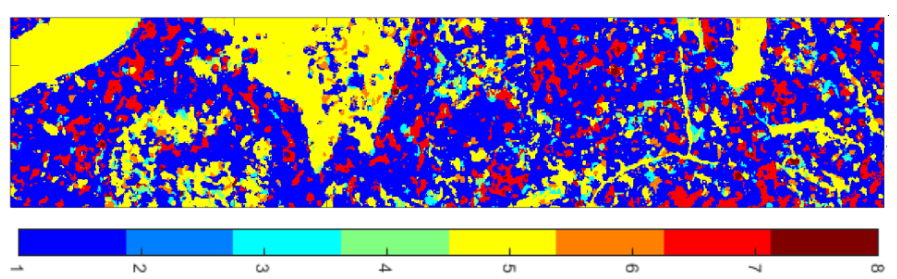

Figure 9. Paracou P-band airborne dataset: statistical classification map. 
SAR Synthetic Aperture Radar

PolSAR Polarimetric Synthetic Aperture Radar

ICTD Incoherent Target Decomposition

ICA Independent Component Analysis

PCA Principal Component Analysis

TSVM Target Scattering Vector Model

MMSE Minimum Mean Square Error

\section{References}

1. Touzi, R. Target scattering decomposition in terms of roll-invariant target parameters. IEEE Transactions on Geoscience and Remote Sensing 2007, 45, 73-84.

2. Vasile, G. Imagerie radar à synthèse d'ouverture interférométrique et polarimétrique. Application au suivi des glaciers alpins. PhD thesis, Université de Savoie, France, 2007.

3. Besic, N.; Vasile, G.; Chanussot, J.; Stankovic, S.; Dedieu, J.; d’Urso, G.; Boldo, D.; Ovarlez, J. Dry Snow Backscattering Sensitivity on Density Change for SWE Estimation. Proceedings of the IEEE International Geoscience and Remote Sensing Symposium, Munchen, Germany, 2012, pp. 4418-4421.

4. Cameron, W.L.; Leung, L.K. Feature motivated polarization scattering matrix decomposition. Proceedings of the IEEE International Radar Conference, Arlington, USA, 1990, pp. 549-557.

5. Vasile, G.; Trouvé, E.; Lee, J.S.; Buzuloiu, V. Intensity-driven-adaptive-neighborhood technique for POLSAR parameters estimation. Proceedings of the IEEE International Geoscience and Remote Sensing Symposium, Seoul, Korea, 2005, Vol. 8, pp. 5509-5512.

6. Cloude, S.R.; Pottier, E. A review of target decomposition theorems in radar polarimetry. IEEE Transactions on Geoscience and Remote Sensing 1996, 32, 498-518.

7. Touzi, R. Speckle effect on polarimetric target scattering decomposition of SAR imagery. Canadian Journal of Remote Sensing 2007, 33, 60-68.

8. Besic, N.; Vasile, G.; Chanussot, J.; Stankovic, S. Polarimetric Incoherent Target Decomposition by Means of Independent Component Analysis. IEEE Transactions on Geoscience and Remote Sensing 2015, 53, 1236-1247.

9. Pralon, L.; Vasile, G.; Dalla-Mura, M.; Chanussot, J.; Besic, N. Evaluation of ICA-Based ICTD for PolSAR Data Analysis Using a Sliding Window Approach: convergence Rate, Gaussian Sources, and Spatial Correlation. IEEE Transactions on Geoscience and Remote Sensing 2016, 54, 4262-4271.

10. Pralon, L.; Vasile, G.; Dalla-Mura, M.; Chanussot, J. Evaluation of the New Information in the H/ $\alpha$ Feature Space Provided by ICA in PolSAR Data Analysis. IEEE Transactions on Geoscience and Remote Sensing 2017, 55, 6893-6909.

11. Cloude, S.R.; Pottier, E. An entropy based classification scheme for land applications of polarimetric SAR. IEEE Transactions on Geoscience and Remote Sensing 1997, 35, 68-78.

12. Vasile, G. Independent Component Analysis Based Incoherent Target Decompositions for Polarimetric SAR Data - Practical Aspects. Proceedings of IEEE International Geoscience and Remote Sensing Symposium, Valencia, Spain, 2018, pp. 5859-5862.

13. Vasile, G. On ICA based ICTD classification of POLSAR data. Proceedings of IEEE International Geoscience and Remote Sensing Symposium, Yokohama, Japan, 2019, pp. 5129-5132.

14. Lee, J.S.; Ainsworth, T.L.; Wang, Y.; Chen, K.S. Polarimetric SAR Speckle Filtering and the Extended Sigma Filter. IEEE Transactions on Geoscience and Remote Sensing 2015, 53, 1150-1160.

15. Vasile, G.; Pascal, F.; Ovarlez, J.P.; Formont, P.; Gay, M. Optimal Parameter Estimation in Heterogeneous Clutter for High Resolution Polarimetric SAR Data. IEEE Geoscience and Remote Sensing Letters 2011, 8, 1046-1050.

16. Bhatia, R.; Holbrook, J. Riemannian geometry and matrix geometric means. Linear Algebra and its applications 2005, 413, 594-618.

17. Kostinski, A.; Boerner, W.M. On foundations of radar polarimetry. IEEE Transactions on Antennas and Propagation 1986, 34, 1395-1404.

18. Formont, P.; Pascal, F.; Vasile, G.; Ovarlez, J.P.; Ferro-Famil, L. Statistical Classification for Heterogeneous Polarimetric SAR Images. IEEE Journal of Selected Topics in Signal Processing 2011, 5, 398-407. 
13 of 13

19. Formont, P.; Ovarlez, J.P.; Pascal, F.; Vasile, G.; Ferro-Famil, L. On the Extension of the Product Model in POLSAR Processing for Unsupervised Classification Using Information Geometry of Covariance Matrices. Proceedings of the IEEE International Geoscience and Remote Sensing Symposium, Vancouver, Canada, 2011, pp. 1361-1364. 